\title{
Hopping and phase shifts in noisy periodically driven bistable systems
}

\author{
Peter Jung, Peter Hänggi \\ Institut für Physik, Universität Augsburg, Memminger Strasse 6, W-8900 Augsburg, Federal Republic of Germany
}

Received: 2 July 1992

\begin{abstract}
We consider a periodically driven bistable system in the presence of fluctuations. In a number of recent papers it has been shown that the amplitude of the response of the noisy system to periodic modulations exhibits stochastic resonance, i.e. a resonance-like behavior as a function of the noise intensity. In this paper, we consider the phase shift between the response and the periodic driving. For weak periodic driving, the phase shift also shows a resonance like behaviour as a function of the noise strength, but this effect is shown to be of different origin than the one responsible for stochastic resonance. Furthermore, the phase shift is demonstrated to exhibit a resonance-like behavior as a function of the driving frequency.
\end{abstract}

\section{Introduction}

In the last years, periodically driven bistable systems in the presence of noise have been studied in the context of stochastic resonance [1-8]. Stochastic resonance is characterized by a resonance like behavior of the response amplitude of the noisy bistable system on periodic forcing as a function of the noise strength. It occurs, when the mean first passage time out of one basin of attraction is half the period of the driving frequency, i.e. when a statistical time scale agrees with a deterministic time scale. In fact, the modulation is even amplified by pumping energy from the noise source into the signal [2]. This effect carries great potential for technological applications such as for instance for the design of detectors. It possibly also plays a role in the information encoding process of the brain out of neuron spike sequences [9].

In this paper, we consider the phase shift of the response of a noisy bistable system to periodic modulations. In case of a dynamical resonance the phase shift is zero for vanishing driving frequency and increases in the resonance region to reach $\pi$ for large frequencies. In the context of stochastic resonance (a statistical resonance), the question of the corresponding phase shifts has been first addressed by Gammaitoni et al. [10] and later in a conflicting paper by Dykman et al. [11]. The paper by Gammaitoni et al. is based on a two-state filtered bistable stochastic process with periodic forcing, i.e. it neglects all relaxation processes within the basins of attraction. The paper by Dykman et al. is solely based on linear response theory. In [11], the authors state, in contrast to [10], that stochastic resonance is connected with a resonance like phase shift. Our analysis in this paper is based on a full numerical solution without any restrictions such as small modulation strength or/and small driving frequencies or/and two-state filtering as well as on a careful linear response study.

The paper is organized as follows: in Sect. 2, we introduce the model and the basic concepts. In the third section we present numerical results for the phase shifts and a careful study of the phase shifts in the context of stochastic resonance. Since our numerical results are not restricted to small modulation strengths, we can study our model in regimes where stochastic resonance is present and in regimes where it is not. We show in detail that the phase shift is not related to stochastic resonance, but exhibits a behavior which is interesting by itself. In the Sect. 4, we compare our numerical results both with analytical results obtained for small modulation strength and with a two-state approximation [2].

\section{The model and basic concepts}

An archetype bistable model is the overdamped motion in a double well potential $V(x)=b x^{4} / 4-a x^{2} / 2$. The system is disturbed by Gaussian white noise $\xi(t)$ with zero mean, i.e.

$$
\begin{aligned}
& \langle\xi(t)\rangle=0 \\
& \left\langle\xi(t) \xi\left(t^{\prime}\right)\right\rangle=2 D \delta\left(t-t^{\prime}\right)
\end{aligned}
$$

and additional periodic modulations $A \sin (\Omega t+\varphi)$. The statistical distribution of the general unknown initial 
phase of the modulator $\varphi$ is assumed to be uniform over the interval $[0,2 \pi]$. The corresponding Fokker-Planck equation in dimensionless variables $(x \rightarrow x \sqrt{b / a}, t \rightarrow a t$, $\left.A \rightarrow A \sqrt{b / a^{3}}, D \rightarrow D b / a^{2}\right)$ reads

$$
\frac{\partial}{\partial t} P(x, t ; \varphi)=\left(\mathbf{L}_{0}+\mathbf{L}_{1}(t)\right) P(x, t ; \varphi),
$$

with the operators

$\mathbf{L}_{0}=-\frac{\partial}{\partial x}\left(x-x^{3}\right)+D \frac{\partial^{2}}{\partial x^{2}}$,

and

$\mathbf{L}_{1}=-A \frac{\partial}{\partial x} \sin (\Omega t+\varphi)$.

The Fokker-Planck equation describes a Markovian nonstationary stochastic process. One of the most significant characteristics of this system is that it approaches for large times a time-periodic probability density $P_{\text {as }}(x, t ; \varphi)$ $[12]$ and that the phase averaged correlation function $\left\langle\left\langle x(t) x\left(t^{\prime}\right)\right\rangle_{\xi}\right\rangle_{\varphi}$, being a function of the time-difference $t-t^{\prime}$ only, does not decay to zero, but approaches a time periodic function [12]. The spectral density, obtained as the Fourier transform of the phase averaged correlation function (a consequence of an extended version of the Wiener Khintchine theorem [13]) in turn shows $\delta$-spikes at odd multiples of the driving frequencies [12]. The weight of the $\delta$-spike at the driving frequency $\Omega$, being proportional to the coherent output power $P_{c}$ of the system, is given by the absolute square of the Fourier coefficient of the first moment of the asymptotic large time probability density at frequency $\Omega$, i.e. [7]

$P_{c}=16 \pi^{2}\left|M_{1}^{2}\right|$,

where

$\langle x(t) ; \varphi\rangle=\sum_{n=-\infty}^{\infty} M_{n} \exp (\operatorname{in}(\Omega t+\varphi))$.

This result is an exact result and not restricted to small modulation strengths or/and small driving frequencies. The absolute value of $M_{1}$ enters the computation of the coherent output power (5) or equivalently, the spectral signal amplification at the driving frequency, i.e. [7]

$\eta=\frac{P_{c}}{P_{\text {in }}}=\frac{P_{c}}{4 \pi^{2} A^{2}}=4 \frac{\left|M_{1}\right|^{2}}{A^{2}}$.

The phase $\Delta$ of $M_{1}$ determines the phase shift between the input signal $A \sin (\Omega t+\varphi)$ and the Fourier component of the output signal at the driving frequency $\Omega$ by

$\Delta=\frac{\pi}{2}-\arctan \left(\frac{\operatorname{Im}\left(M_{1}\right)}{\operatorname{Re}\left(M_{1}\right)}\right)$.

We like to emphasize here that contrary to the claims raised in [11] the phase shift $\Delta$ does not occur in the phase averaged correlation function $\left\langle\left\langle x(t) x\left(t^{\prime}\right)\right\rangle_{\xi}\right\rangle_{\varphi}$.
One can show, in fact, that for large times, the phase averaged correlation function is given by [12]

$\left\langle\left\langle x(t) x\left(t^{\prime}\right)\right\rangle_{\xi}\right\rangle_{\varphi}=2 \sum_{n=1}^{\infty}\left|M_{n}\right|^{2} \cos \left[n \Omega\left(t-t^{\prime}\right)\right]$.

This result has been established further by use of analog simulations [3]. It has been shown in [7] that the spectral amplification $\eta$ in (7) shows - similar to the signal/noise ratio $[1,2]$ - stochastic resonance, i.e. it increases with increasing noise strength to reach for small driving frequencies a peak value much larger than unity and then decreases again upon increasing the noise strength further. The central object in this paper is the behavior of the phase shift in the regime, when the amplification shows stochastic resonance.

\section{Numerical results for the phase shift}

Using the same technique as in [14], we obtain the complex Fourier coefficients of the asymptotic mean value $M_{n}$ in terms of matrix continued fractions [15]. The numerical errors of the results are within line thickness. In Fig. 1 we have compared the amplification $\eta$ with the phase shift $\Delta$ as a function of the noise strength for $A=0.1$ and $\Omega=0.1$. The amplification shows a resonance-like peak at $D \approx 0.15$. In the region around this peak, the phase shift shows monotonic decrease for increasing noise strengths. For smaller values of the noise (about 50\% smaller), however, the phase shift also shows a peak (see also [11]). In this regime, the amplification shows monotonic behavior. The question which raises at this point is, to what extend the peak of the phase shift is related to stochastic resonance, being responsible for the peak in the spectral amplification. Calculating the amplitude response and the phase shift for larger modulation strengths (a regime which cannot be studied within linear response theory), we find that the peak of the phase shift has disappeared while the resonance in the amplification is still present. In Fig. 2, we show the same quantities as in Fig. 1, but with $A=0.4$. This results implies that stochastic resonance is not necessarily connected with a peak in the phase shift.

It is also interesting to look at the dependence of the phase shift on the driving frequency. The amplification shows (see Fig. 3) monotonous decrease for increasing frequencies. In contrast, the phase shift shows for small noise strength a sharp peak at a small frequency. For further increasing frequency it reaches a minimum and then increases again. As discussed in the next section, the reason for this behavior is the competition between intraand interwell motion. For small frequencies, the response of the system to periodic modulation is dominated by hopping dynamics, while for larger frequencies, the system response is dominated by relaxation motion within the potential wells. For increasing noise strength, the peak shifts to larger values of the frequency and becomes smoother (see Fig. 4a, b). For increasing modulation strength $A$, the maximum of the phase shift is also shifted 

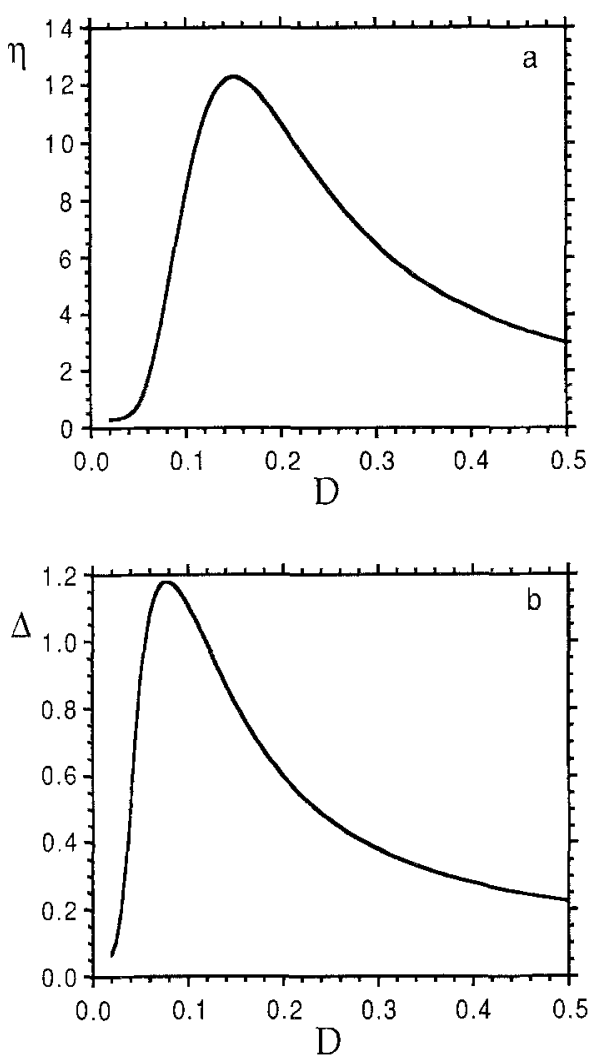

Fig. 1. The spectral amplification $a$ is compared for $\Omega=0.1$ and $A=0.1$ as a function of the noise strength with the phase shift $\mathbf{b}$
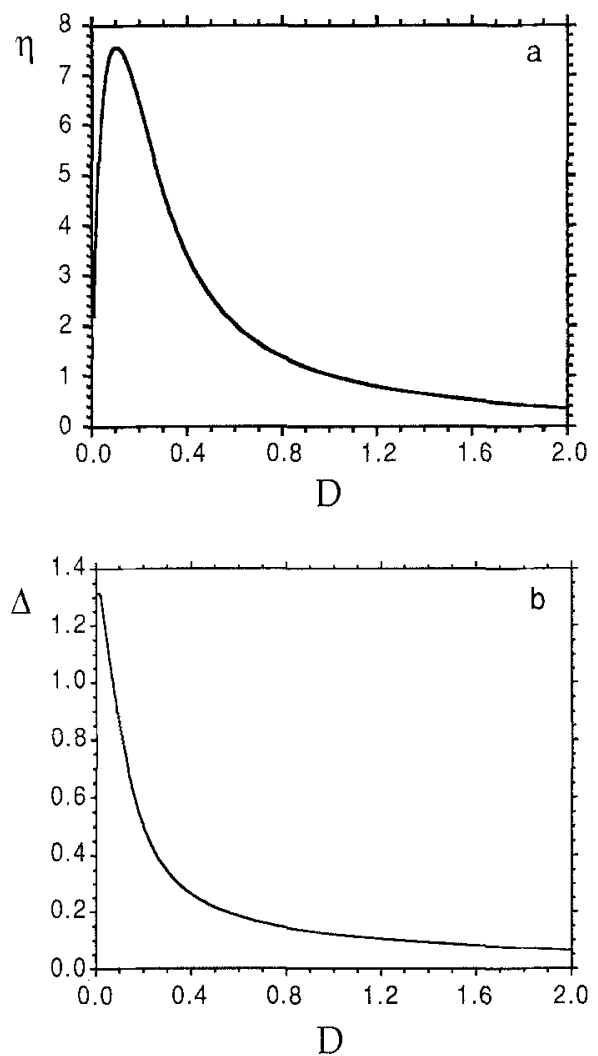

Fig. 2. The spectral amplification a is compared for $\Omega=0.1$ and $A=0.4$ as a function of the noise strength with the phase shift $b$
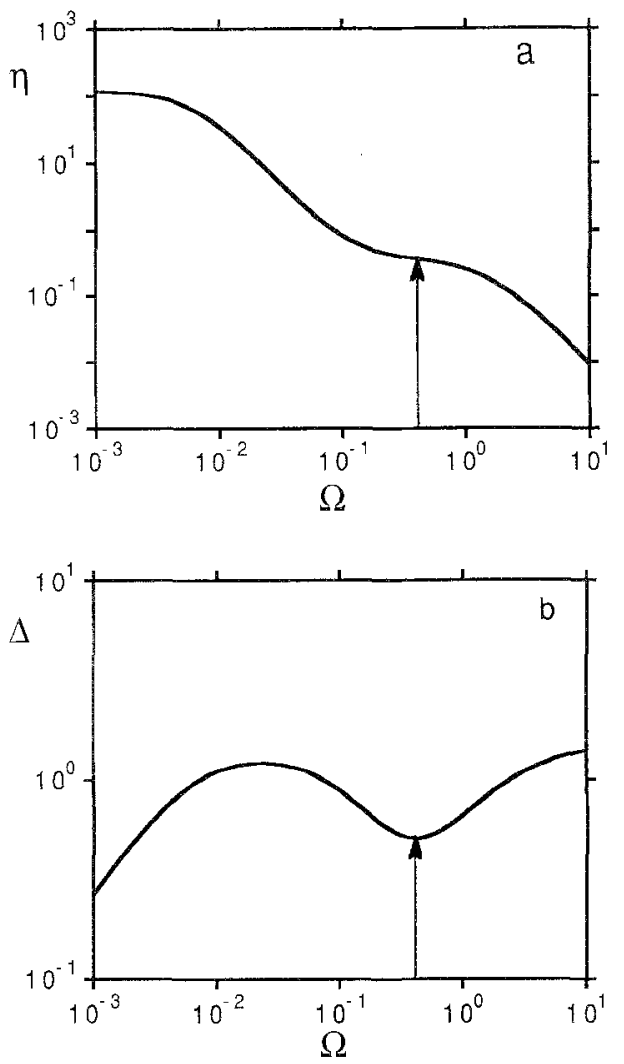

Fig. 3. The spectral amplification $\mathbf{a}$ is compared for $D=0.05$ and $A=0.1$ as a function of the frequency $\Omega$ with the phase shift $\mathbf{b}$
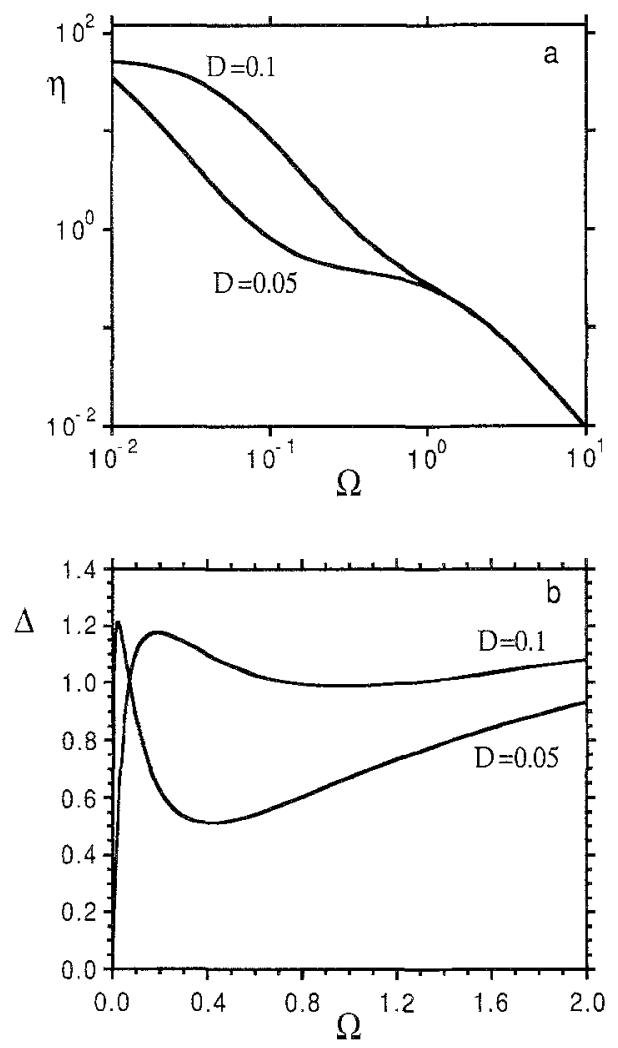

Fig. 4. The spectral amplification $\mathbf{a}$ and the phase shift $\mathbf{b}$ is shown for $D=0.1$ and $D=0.05$ at $A=0.1$ as a function of the frequency $\Omega$ 

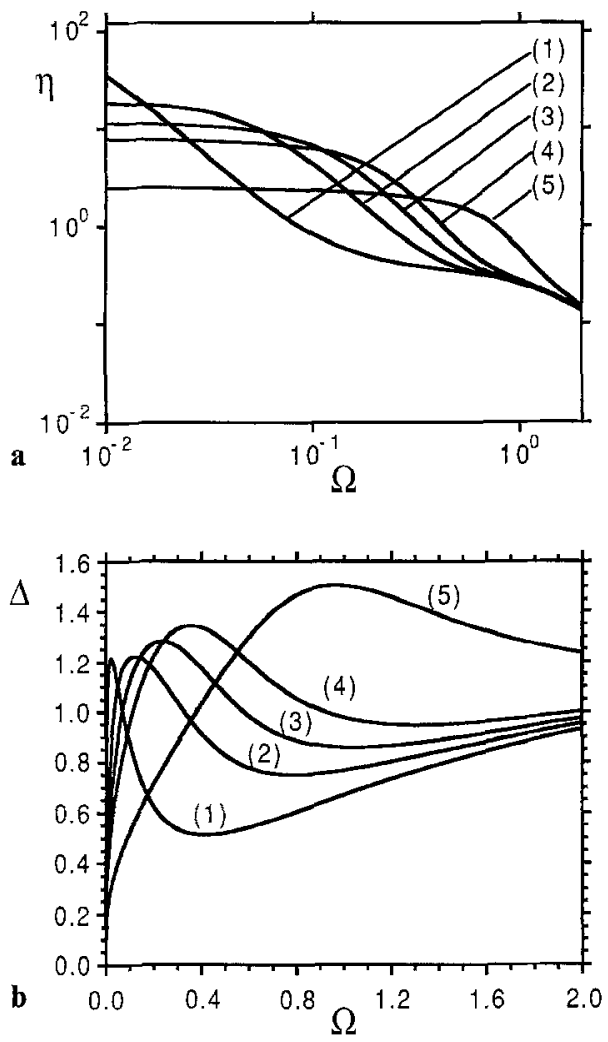

Fig. 5. The spectral amplification a and the phase shift $\mathbf{b}$ is shown at $D=0.05$ for $A=0.1(1), A=0.3(2), A=0.4(3), A=0.5(4)$ and $A=1(5)$ as a function of the driving frequency

to larger values of the driving frequency (Fig. 5). It is interesting to note that the amplitude response shows a "shoulder" at those values of the frequency, where the phase shift exhibits a relative minimum (note the different scales in Fig. 4).

\section{Linear response approach}

Considering the second part of the Fokker-Planck operator $\mathbf{L}_{1}$ as small compared to the first term $\mathbf{L}_{0}$, we can apply linear response approximation [16]. The response of the system is then described as a convolution integral

$\langle x(t) ; \varphi\rangle=A \int_{-\infty}^{\infty} \mathrm{R}\left(t-t^{\prime}\right) \sin \left(\Omega t^{\prime}+\varphi\right) \mathrm{d} t^{\prime}$,

with the response function

$R(\tau)=\left\{\begin{array}{cl}-\int_{-\infty}^{\infty} x \exp \left(L_{0} \tau\right) \frac{\partial}{\partial x} P_{\mathrm{St}}(x) \mathrm{d} x & \text { for } \tau>0 \\ 0 & \text { for } \tau<0,\end{array}\right.$

and $P_{\mathrm{St}}(x)$ being the stationary probability of the nondriven system $(A=0)$. Using the fluctuation theorem, the response function is related to the correlation function by $[16]$

$\dot{K}_{x x}(\tau)=-D R(\tau)$.
The correlation function is approximated by the sum of two exponentials, i.e.

$K_{x x}(\tau)=g_{1} \exp \left(-\lambda_{\min } \tau\right)+g_{2} \exp (-\alpha \tau)$,

where the first one describes hopping between the stable states and the second one stochastic motion within one region of attraction (intra-well motion) (for our particular system: $\alpha=2$ ). For (13) to be valid, we have to require a sufficiently large time scale separation between hopping and intra-well motion, i.e. a small noise strength $D$. The yet unknown statistical weights $g_{1}$ and $g_{2}$, can be determined from the correlation function and its derivative at $\tau=0$, yielding

$g_{2}=\frac{\lambda_{\min }\left\langle x^{2}\right\rangle_{\mathrm{st}}}{\lambda_{\min }-\alpha}+\frac{\left\langle x^{2}\right\rangle_{\mathrm{st}}-\left\langle x^{4}\right\rangle_{\mathrm{st}}}{\lambda_{\min }-\alpha}$

$g_{1}=\left\langle x^{2}\right\rangle_{\mathrm{st}}-g_{2}$,

where

$\left\langle x^{2 n}\right\rangle=\sqrt{2 D} \frac{\Gamma(n+1 / 2)}{\Gamma(1 / 2)} \frac{D_{-n-1 / 2}(-1 / \sqrt{2 D})}{D_{-1 / 2}(-1 / \sqrt{2 D})}$,

with $D_{r}(x)$ being parabolic cylinder functions [17]. The susceptibility, i.e. the Fourier transform of the response functions thus reads

$$
\begin{aligned}
\hat{R}(\omega)= & \frac{g_{1} \lambda_{\min }^{2}}{D} \frac{1}{\lambda_{\min }^{2}+\omega^{2}}+\frac{g_{2} \alpha^{2}}{D} \frac{1}{\alpha^{2}+\omega^{2}} \\
& -\frac{i \omega}{D}\left(\frac{g_{1} \lambda_{\min }}{\lambda_{\min }^{2}+\omega^{2}}+\frac{g_{2} \alpha}{\alpha^{2}+\omega^{2}}\right) .
\end{aligned}
$$

Using (10) and (6), the Fourier coefficient $M_{1}$ of the mean value $\langle x(t) ; \varphi\rangle$ is obtained as

$M_{\mathrm{I}}=\frac{A}{2 i} \hat{R}(\Omega)=\frac{A}{2}|\hat{R}(\Omega)| \exp \left[i \psi(\Omega)-i \frac{\pi}{2}\right]$

with $\psi(\Omega)$, being the phase of the response function $\hat{R}(\Omega)$. This yields for the spectral amplification $\eta$

$$
\begin{aligned}
\eta= & 4 \frac{\left|M_{1}\right|^{2}}{A^{2}}=|\hat{R}(\Omega)|^{2}=\frac{1}{D^{2}}\left[\frac{g_{1}^{2} \lambda_{\min }^{2}}{\lambda_{\min }^{2}+\Omega^{2}}+\frac{g_{2}^{2} \alpha^{2}}{\alpha^{2}+\Omega^{2}}\right. \\
& \left.+\frac{2 g_{1} g_{2} \alpha \lambda_{\min }\left(\alpha \lambda_{\min }+\Omega^{2}\right)}{\left(\lambda_{\min }^{2}+\Omega^{2}\right)\left(\alpha^{2}+\Omega^{2}\right)}\right],
\end{aligned}
$$

and the phase shift

$$
\Delta(\Omega)=\arctan \frac{\operatorname{Im}(\hat{R}(\Omega))}{\operatorname{Re}(\hat{R}(\Omega))}-\frac{\pi x}{2} .
$$

In the weak noise limit $D \rightarrow 0$, the statistical weights are obtained by expanding the stationary mean values in $(14,15)$ according to $[17]$

$$
\begin{aligned}
& D_{-n-1 / 2}(-1 / \sqrt{2 D}) \stackrel{D \rightarrow 0}{\longrightarrow} \frac{\sqrt{2 \pi}}{\Gamma(n+1 / 2)} \\
& \quad \times \exp \left(\frac{1}{8 D}\right)(2 D)^{1 / 4-n / 2}
\end{aligned}
$$




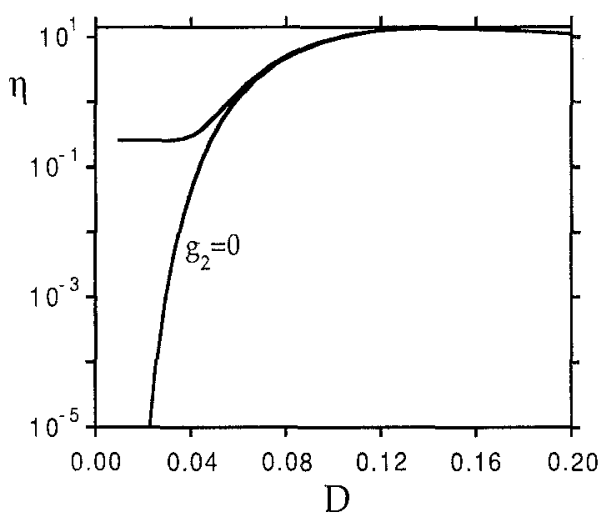

Fig. 6. The spectral amplification is shown in linear response approximation including intra-well motion (a) and for the pure hopping process

as

$g_{1} \approx 1-\left(1+\frac{1}{\alpha}\right) D$

$g_{2} \approx \frac{D}{\alpha}$.

The amplification $\eta$ is then given for a finite driving frequency

$\eta \stackrel{D \rightarrow 0, \Omega: \text { fixed }}{\longrightarrow} \frac{1}{\alpha^{2}+\Omega^{2}}$.

This value agrees very well with the numerical results presented in the last section for small modulation strength. The hopping process does not contribute to the response of the system to periodic forcing in the weak noise limit. To find out the value of the noise strength where the hopping mechanism becomes important, we have compared in Fig. 6 the amplification in (18) with the amplification obtained by neglecting intra-well motion (i.e. $g_{2}=0$ ). Starting from zero noise, the agreement is very poor. When the phase shift starts to increase (see Figs. 1b, $2 \mathrm{~b}$ ) to reach its maximum, the curves approach each other quickly. Thus, the hopping process becomes dominant, when the phase shift approaches its peak (or vice verse).

For the phase shift we find for small noise strength

$\Delta=\arctan \left(\frac{\frac{\Omega}{D} \frac{\lambda_{\min }}{\lambda_{\text {min }}^{2}+\Omega^{2}}+\frac{\Omega}{\alpha^{2}+\Omega^{2}}}{\frac{1}{D} \frac{\lambda_{\min }^{2}}{\lambda_{\min }^{2}+\Omega^{2}}+\frac{\alpha}{\alpha^{2}+\Omega^{2}}}\right)$.

For finite driving frequency, we find in the limit $D \rightarrow 0$

$\Delta=\arctan \frac{\Omega}{\alpha}$,

i.e. a finite value which is determined by the relaxation motion within the wells. With small but increasing noise, the first terms in the nominator and denominator in (23) are the leading terms and the (hopping dominated) phase shift is then obtained as (equivalent with two-state model [2])

$\Delta \approx \arctan \frac{\Omega}{\lambda_{\min }}$.

Since $\lambda_{\min }$ is a small quantity, i.e. $\lambda_{\min } \ll \alpha$, the phase shift in the hopping regime is much larger than in the regime, dominated by intra well motion. We find that the increase in the phase shift for small noise strength is due to the turnover between the regime which is dominated by intrawell motion and that controlled by hopping motion. The peak of the amplification, however, lies well within the hopping dominated regime.

For finite but small noise, the phase shift reads in the adiabatic limit

$\Delta \stackrel{\Omega \rightarrow 0}{\longrightarrow} \arctan \frac{\Omega}{\lambda_{\min }} \approx \frac{\Omega}{\lambda_{\min }} \propto \Omega \exp \left(\frac{1}{4 D}\right)$,

whereas for large frequencies one finds from (24)

$\Delta \approx \arctan \frac{\Omega}{\alpha}$.

Equation (26) explains the large slope of the phase shift as a function of the frequency for small frequencies, observed in our numerical study (see Fig. 4b). Both limits $(26,27)$ agree well with the numerical data for small modulation strength.

\section{Conclusions}

We have studied the phase shift of the response of a noisy bistable system subject to periodic forcing. The phase shift shows for weak modulation a resonance like behavior as a function of the noise strength (c.f. Fig. 1b). The maximum of this resonance like curve does not coincide with the peak of the spectral amplification $\eta$. There are even regimes, where the phase shift does not show a peak (c.f. Fig. 2b), in contrast to the amplification $\eta$. We have identified intra-well motion dominated and hopping dominated regimes. The peak of the phase shift for small driving reflects the turnover between both regimes, while the stochastic resonance peak of the amplitude response lies well within the hopping dominated regime. In other words, stochastic resonance is a pure synchronization effect of a hopping motion with an external signal (acting as a "clock"), while the peak of the phase shift is due to competition between motion on a global and a local time scale; both effects are of different physical origins.

We have found further that the phase shift and the response amplitude show an interesting behavior as a function of the driving frequency $\Omega$. The amplification exhibits a "shoulder-like" shape around the value of the frequency, where the phase shift shows a minimum. For increasing noise strength - as well as for increasing modulation strength - the shoulder of the amplification and the minimum of the phase shift both vanish. 
The validity of the linear response theory has been shown to be very restricted. It fails to describe the disappearance of the peak of the phase shift (large driving amplitudes). Also, it cannot account for the dependence of the peaks of the phase shift on the modulation strength (c.f. Fig. 5). We have further shown in Fig. 6 that the inclusion of intra-well motion correctly reproduces the phase shift maximum for sufficiently small modulation (see also [11]), but does not yield the relevant corrections to the spectral amplification $\eta$ around its maximum. The disagreement of numerical data with linear response results for the response amplitude around the stochastic resonance peak, pointed out in [7], are thus, contrary to the claims raised in [11], due to the failure of linear response theory.

We wish to thank Frank Moss and Luca Gammaitoni for useful discussions. This work has been supported by Stiftung Volkswagenwerk, NATO Grant No. 0770/85.

\section{References}

1. McNamara, B., Wiesenfeld, K., Roy, R.: Phys. Rev. Lett. 60, 2626 (1988)

2. MeNamara, B., Wiesenfeld, K.: Phys. Rev. A39, 4854 (1989)

3. Gammaitoni, L., Marchesoni, F., Menichella-Saetta, E., Santucci, S.: Phys. Rev. Lett. 62, 349 (1989)

4. Presilla, C., Marchesoni, F., Gammaitoni, L.: Phys. Rev. A40, 21.05 (1989)

5. Debnath, G., Zhou, T., Moss, F.: Phys. Rev. A39, 4324 (1989)

6. Zhou, T., Moss, F.: Phys. Rev. A41, 4255 (1990)

7. Jung, P., Hänggi, P.: Phys. Rev. A44, 8032 (1991)

8. Zhou, T., Moss, F., Jung, P.: Phys. Rev. A42, 3161 (1990)

9. Longtin, A., Bulsara, A.R., Moss, F.: Phys. Rev. Lett. 67, 656 (1991)

10. Gammaitoni, L., Marchesoni, F., Martinelli, M., Pardi, L., Santucci, S.: Phys. Lett. A158, 449 (1991)

11. Dykman, M.I., Mannella, R., McClintock, P.V.E., Stocks, N.G.: Phys. Rev. Lett. 18, 2985 (1992)

12. Jung, P., Hänggi, P.: Europhys. Lett. 8, 505 (1989)

13. Jung, P.: Phys. Rep. (submitted for publication)

14. Jung, P.: Z. Phys. B - Condensed Matter 76, 521 (1989)

15. Risken, H. (ed.): The Fokker-Planck equation. Springer Series in Synergetics, Vol. 18. Berlin, Heidelberg, New York: Springer 1984

16. Hänggi, P., Thomas, H.: Phys. Rep. 88, 207 (1982)

17. Abramowitz, M., Stegun, I. (eds.): Handbook of mathematical functions. Sect. 19; 19.3.8, 19.8.2. Dover Publications 1963 